\section{$\underset{\text { hommes migrations }}{\text { \& mions }}$}

\section{Hommes \& migrations}

Revue française de référence sur les dynamiques

migratoires

$1319 \mid 2017$

Réfugiés et migrants au Liban

\title{
Angelo Donati
}

\section{Christiane Garnero Morena}

\section{QpenEdition \\ Journals}

\section{Édition électronique}

URL : http://journals.openedition.org/hommesmigrations/3993

DOI : ERREUR PDO dans /localdata/www-bin/Core/Core/Db/Db.class.php L.34 : SQLSTATE[HY000]

[2006] MySQL server has gone away

ISSN : 2262-3353

Éditeur

Musée national de l'histoire de l'immigration

\section{Édition imprimée}

Date de publication : 1 octobre 2017

Pagination : 144-147

ISBN : 978-2-919040-39-1

ISSN : 1142-852X

Référence électronique

Christiane Garnero Morena, «Angelo Donati », Hommes \& migrations [En ligne], 1319 | 2017, mis en ligne le 01 octobre 2017, consulté le 08 janvier 2021. URL : http://journals.openedition.org/ hommesmigrations/3993 ; DOI : https://doi.org/ERREUR PDO dans/localdata/www-bin/Core/Core/ Db/Db.class.php L.34 : SQLSTATE[HY000] [2006] MySQL server has gone away 


\section{ITALIANITÉ}

\section{ANGELO DONATI}

Par CHRISTIANE GARNERO MORENA, géographe et historienne.

1 ssu d'une ancienne famille de la bourgeoisie juive de Modène, Angelo Donati est né dans cette ville du nord de l'Italie en $1885^{1}$. Rien ne laissait présager l'existence hors du commun qui fut la sienne. Sa vie de jeune homme débute selon les schémas traditionnels de son milieu social : des études juridiques, une formation aux métiers de la fiance à Milan et Turin, quand arrive la Première Guerre mondiale. L'Italie entre en guerre en 1915 au coté de la Triple Alliance ${ }^{2}$. Angelo Donati rejoint le front. Incorporé dans un premier temps dans un régiment d'infanterie, il se fera remarquer par la suite dans l'aviation, obtient un grade de lieutenant et devient agent de liaison avec l'armée française. Premier événement qui va donner une orientation à sa vie, il siège pour l'Italie à la Commission d'armistice en France, en 1918-1919.

\section{Paris, 1919-1939}

Angelo Donati s'installe à Paris dès 1919. Il mène une carrière d'homme d'affaires mais aussi de diplomate. De 1925 à 1932, il sera consul général de la République de Saint-Marin et, en 1932, il devient président de la Chambre de commerce italienne et directeur du Banco di Roma, ainsi que président de la Croix Rouge italienne en France et administrateur de nombreuses sociétés. En 1936, il reçoit la
Légion d'honneur. II rencontre Haim Weismann³ avec qui il restera en contact et va aider financièrement l'implantation juive en Palestine.

Angelo Donati va se marier une première fois avec une aristocrate égyptienne proche du roi Farouk. Le couple s'installe rue de Berry, appartement qu'Angelo Donati conservera toute sa vie. Cette union va devenir pour lui comme une sorte de tremplin lui ouvrant les portes du tout Paris de l'entre-deux-guerres ${ }^{4}$. Son épouse l'initie aux pratiques protocolaires de la cour d'Égypte. Angelo Donati devient un homme mondain en vue. Mais son épouse à laquelle il était très attaché décède brutalement des suites d'un accident de voiture à la fin des années 1920.

II se remarie quelques années plus tard avec une très belle femme d'origine hongroise. Cette dernière deviendra célèbre sur les Champs-Élysées qu'elle parcourt en tenant en laisse ses superbes chiens Barzoïs. Mais le couple se sépare, la jeune femme quitte la France pour les États-Unis en 1939. En septembre 1939, le décret d'Albert Sarrault visant à l'internement des juifs étrangers contraint Angelo Donati à quitter Paris. Il se déplace dans la zone libre, y croise pour la première fois Giuseppe Saragat 5 , et arrive à Nice. II s'y installe, prend un appartement sur la Promenade des anglais ${ }^{6}$ et essaie de continuer ses activités autant que possible. 
Angelo Donati à son bureau dans les années 1920. (c) MARIANNE SPIER-DONATI.

\section{Nice}

Certes, Nice est située en zone libre, mais les milices collaborationnistes du gouvernement de Vichy effectuent de nombreuses rafles, tout particulièrement parmi les juifs étrangers qui, après de longues pérégrinations à travers l'Europe, sont arrivés dans cette ville de tradition cosmopolite. Ils ont fuis les promulgations de lois raciales et l'avancée des troupes hitlériennes. Ils essaient de survivre sur la Riviera où ils sont souvent assignés à résidence.

En 1942, la vie d'Angelo Donati va croiser le destin de la famille Spier. Marianne et Rolf Spier et leurs parents: Carl et Hilde. Après avoir quittés l'Allemagne, la famille Spier arrive en France. Les Spier ${ }^{7}$ sont d'abord internés dans le camp de Gurs ${ }^{8}$, puis assignés à résidence à Cap d'Ail, petite ville entre Monaco et Nice. La famille reçoit la visite d'une cousine de madame Spier, Lise Klein et de son mari Piero Sacerdoti. Ces derniers laissent à tout hasard un numéro de téléphone qui pourrait être utile : le numéro d'Angelo Donati, un cousin de Piero Sacerdoti.
Quelques jours après cette visite, la famille Spier est arrêtée par la police française et conduite à la caserne de l'Auvare à Nice. Comprenant que la situation est très grave, les parents Spier, pour sauver leurs enfants 9 , se font hospitaliser. Marianne raconte qu'elle a vu passer sa mère sur une civière en silence, échange de regard, le dernier.

Les enfants sont conduits dans les bureaux de I'Union générale des israélites de France $\left(\mathrm{UGIF}^{10}\right)$. On leur demande s'ils ont un point de chute. Après un premier appel à des voisins suivi par un refus, Marianne donne un numéro de téléphone. Elle avait toujours sur elle, caché, un petit carnet remis par sa mère avec des numéros et informations utiles. Le numéro de téléphone que donne Marianne est celui d'Angelo Donati. Ce dernier, absent, ne prend pas directement l'appel. Mais, dès son retour chez lui, informé, il se précipite aux bureaux de l'UGIF et prend les enfants Spier avec lui. Il les conduit quelques jours chez des amis, le temps d'aménager son appartement de la Promenade des anglais pour les recevoir chez lui. Le sort des enfants Spier sera désormais lié à Angelo Donati. Il devient « Zio Angelo ».

\section{« Nizza Italiana »}

Le 11 novembre 1942, l'armée italienne, bersaglieri" plumes au vent en tête, occupe Nice. Un air de revanche à l'annexion de 1860 qui n'a toujours pas été complètement acceptée par certains italiens. Angelo Donati entretient avec les autorités fascistes et les militaires hauts gradés de l'armée italienne les meilleures relations. Il est directeur de la banque franco-italienne. Dans l'ombre, son rôle va être capital pour orienter le comportement des

7. Carl Spier était directeur d'usine et Hilde Spier, docteure en philologie allemande, était journaliste. 8. Le camp de Gurs, construit en 1939 pour y regrouper des réfugiés espagnols républicains, deviendra sous le gouvernement de Vichy un camp d'internement pour les juifs étrangers. 9. Les enfants de moins de 16 ans n'étaient pas en théorie déportés. 10. Organisation créée en novembre 1941 à la demande des autorités allemandes. Sa mission : assurer la représentation des juifs auprès des pouvoirs publics, notamment pour les questions d'assistance, de prévoyance et de reclassement social. 11. Les bersaglieri est un corps d'infanterie, qui se caractérise au niveau du costume par un superbe casque plat avec somptueux panache de plumes de coq (Tetrao urugallos) de couleur noire. 


\section{ITALIANITÉ}

autorités fascistes et militaires italiennes. Ces dernières, malgré la présence d'un bureau de l'OVRA'2, adoptent une position de totale indépendance par rapport aux allemands' ${ }^{13}$. D'autant plus que le consul général d'Italie qui vient d'être nommé à Nice par le comte Galeazzo Ciano (ministre des affaires étrangères et gendre de Mussolini), Alberto Calisse, connait depuis les années 1920/30 Angelo Donati, qu'il nomme "porte-parole des milieux juifs ${ }^{14}$ ».

Angelo Donati est plus que jamais actif.II prend en main le sort de la communauté juive, non seulement de Nice mais de toute la zone d'occupation italienne. Avec une grande diplomatie, il entretient non seulement d'excellents rapports avec les autorités italiennes fascistes et les réseaux d'entraide ${ }^{15}$ mais joue également de ses relations avec le Vatican et ainsi établit des contacts avec les Alliés pour préparer son plan.

Marianne et son petit frère sont les enfants de la maison. Ils assistent amusés au défilé des hauts gradés et hiérarques fascistes dans leurs uniformes impeccables. Rolfs'amuse même discrètement avec les armes de services déposées dans le vestibule.

\section{Le plan de Donati}

Début 1943, Donati commence à mettre en place les étapes de son plan incroyable : celui d'évacuer le plus grand nombre de juifs de la zone d'occupation italienne vers l'Afrique du Nord déjà libérée, puis de les conduire en Palestine. Dans un premier temps, il met en place une organisation pour procurer des pièces d'identité. Puis, il trouve les financements et frette 4 paquebots déjà amarrés dans des ports italiens.

8 septembre 1943, le général Eisenhower sans en avertir les autorités italiennes, rend publique l'armistice, qui devait rester sec, signé le 3 septembre avec le maréchal Badoglio' ${ }^{16}$. Pour l'Italie se sera le début des répressions nazies avec la chute de Mussolini et, en France, la fin de la zone libre. À Nice, où plus de 25000 juifs se sont réfugiés, c'est la fin de « ce paradis » comme le dit Serge Klarsfeld. Le terrible Alois Brunner et ses sbires aidés par la milice du gouvernement de Vichy sont libres de se livrer à leur sinistre besogne. La ville devient le théâtre d'une série de rafles qui vont également profondément marquer la population : le regroupement avant déportation par le terrible passage à Drancy se déroule à l'hôtel Excelsior, à deux pas de la gare centrale aux yeux de tous.

Angelo Donati, avec Marianne et Rolf et son major d'homme François Moraldo, se réfugie en Toscane. Il ne peut plus rentrer à Nice où la Gestapo l'attend. Il va avec des membres de sa famille se cacher quelques mois avant de passer en Suisse. Comprenant qu'il ne peut pas entraîner les enfants Spier dans sa fuite, il les confie à François Moraldo. Ce dernier part rejoindre son petit village des montagnes ligures au-dessus de Sanremo, Creppo. Creppo, en 1943, c'est le bout du monde, pas d'électricité, pas de téléphone, pas de T.S.F! «Nous y sommes arrivés à dos de mulet. II n'y avait pas de route", raconte Marianne. Intégrés dans la communauté villageoise, logés dans la famille de

\footnotetext{
12. Police secrète fasciste de 1930 à 1945. 13. Début novembre 1942, le chef d'état major générales armées italiennes - Guido Lo Spinosa - déclare aux Allemands : "Les violences contre les juifs ne sont pas compatibles avec l'honneur de l'armée italienne. " Les juifs seront assignés à résidence et sous protection italienne, ce qui sauvera des centaines qui suivront sur les sentiers des Alpes, l'armée italienne en déroute et trouveront pour beaucoup d'entre eux refuge en Italie. 14. Alberto Calisse va fournir à Angelo Donati tous les faux papiers nécessaires pour la réalisation de son plan. En outre, pour sauver les plus démunis, il va les rassembler dans un camps de résidence forcée pour déjouer les demandes constantes des nazis que leur soient "livrés " ces pauvres fugitifs. Plus d'un millier seront donc conduits dans le village de montagne de l'arrière-pays niçois de Saint-Martin-Vésubie. Mais, après le 8 septembre 1943 , ce sera la fuite vers l'Italie par des sentiers de haute montagne à plus de 2 ooo mètres d'altitude avec l'armée italienne en déroute. Plus de 750 d'entres eux seront cachés chez le montagnards de la région de Coni, car le Piémont est envahi par les troupes hitlériennes. Cet épisode est entre autres relaté dans un livre de Jean Marie Le Clezio : Étoile errante, Paris, Gallimard, 1994. 15. Voir Jean-Louis Panicacci, "Les territoires occupés par les forces armées italiennes, juin 1940-novembre 1942 ”, in Histoire(s) de la Dernière Guerre, n 6, juillet 2010 ; L'Occupation italienne-Sud-Est de la France, juin 1940-septembre 1943, Paris, Presses universitaires de Rennes, 2010. 16. Cet armistice secret est signé avec les alliés dans le petit village de Cassable en Sicile près de Syracuse le 3 septembre 1943.
} 
Angelo Donati entouré de Rolf et Marianne Spier-Donati dans les années 1950. (C) MARIANNE SPIER-DONATI.

François Moraldo, Marianne et Rolf prennent le nom de Spiro, que Marianne pensait italien - en réalité c'est un nom juif. Ils deviennent de petits montagnards, vivant comme les autres enfants du village aux rythmes des saisons.

Angelo Donati passe la fin de la guerre à Montreux et il continue d'agir pour sauver le plus grand nombre possible de ses coreligionnaires.

\section{5}

François Moraldo reçoit une lettre : «Ramènes les enfants à Paris. "Marianne et son petit frère suivent François en train et arrivent à Paris. Ils reprennent, rue de Berri, leur vie d'enfants de la maison avec « Zio Angelo ».

Angelo Donati va, quand à lui, être très impliqué dans la reconstruction du tissu social et des relations entre la France et l'Italie. Il est nommé délégué général de la Croix Rouge italienne. À Paris, il retrouve Giuseppe Saragat qui est ambassadeur en France et se lie d'amitié avec le nonce apostolique : Angelo Roncali, le futur Jean XXIII. II sera, entre autres, très actif pour intercéder en faveur du rapatriement en Italie de prisonniers militaires. Très proche de René Mayer ${ }^{17}$, il participe également à la renaissance des institutions juives en France. En 1953, il devient le chargé d'affaires de la République de Saint-Marin avec rang de ministre plénipotentiaire.

Et il reprend sa vie mondaine. La petite fille de René Mayer, L.A., raconte que, lors d'un dîner chez ses parents, Angelo Donati l'aperçoit avec sa sœur jumelle le nez collé aux vitres qui séparent le salon de la salle à manger.II intercède auprès des parents pour que les petites filles rejoignent les grandes personnes pour le dessert.

Quand arrive la confirmation que les parents de Marianne et Rolf ne reviendront plus, Angelo Donati les adopte. Ils porteront désormais le nom Spier-Donati. Malade depuis quelques années, Angelo Donati s'éteint chez lui le 30 décembre 1960. II repose dans le caveau familial du cimetière juif de Modène où vit encore une partie de sa famille.

Ce parcours de vie hors du commun interpelle. Peut être aimait-il tellement la vie qu'il a trouvé en lui la capacité, malgré les périodes les plus sombres du XXe siècle qu'il a vécues, d'être capable d'imaginer, en stratège de génie mais discret, des projets les plus fous pour le bien du plus grand nombre? Le plan d'Angelo Donati, qui aurait pu devenir une opération encore plus spectaculaire que la fameuse liste de Schindler ne sera pas réalisé et demeure encore aujourd'hui ignoré du grand public.

Finalement, Angelo Donati était un homme du monde, très à l'écoute des autres. Il a traversé avec élégance le siècle. Sans être réellement anonyme, il n'a jamais cherché à médiatiser ses actions. Ce qui comptait pour lui avant tout c'était de faire. II devait avoir un amour immodéré de la vie, de ses vraies valeurs. Il a choisi de vivre selon son cœur. Il portait bien ce prénom d'Angelo : «ange » en italien! 